ANNALES

POLONICI MATHEMATICI

$92.2(2007)$

\title{
Extending o-minimal Fréchet derivatives
}

\author{
by ANDREAS Fischer (Saskatoon)
}

\begin{abstract}
We investigate several extension properties of Fréchet differentiable functions defined on closed sets for o-minimal expansions of real closed fields.
\end{abstract}

1. Introduction. In the present paper, which is inspired by the author's doctoral thesis, and which is motivated by [1], we study extendibility of Fréchet differentiable functions defined on closed sets for o-minimal structures.

In the following, let $R$ denote a fixed real closed field, and let $\mathcal{M}$ be an o-minimal expansion of $R$. Here, "definable" always means "definable with parameters in $\mathcal{M}$ ". For comprehensive references on o-minimality we refer the reader to [5] and [4]; these sources are also suitable for those who are not specifically familiar with o-minimality. Classical examples of o-minimal structures are the semialgebraic structure and the structure consisting of all globally subanalytic sets; for recent examples, see [11], [12], [14], [6], [7] and $[15]$.

Let $R^{n}$ be endowed with the Euclidean $R$-norm $\|\cdot\|$ and the corresponding topology. Note that $R$-norm has the same definition as the norm except that it takes its values in $R$. The notion of Fréchet differentiability extends to arbitrary sets as follows.

Definition 1.1. Let $A \subset R^{n}$. A function $f: A \rightarrow R^{k}$ together with a function $\varphi: A \rightarrow R^{k \times n}$ is called Fréchet differentiable relative to $A$ if

$$
\lim _{A \ni x \rightarrow a} \frac{f(x)-f(a)-\varphi(a)(x-a)}{\|x-a\|}=0, \quad a \in A .
$$

If $f$ and $\varphi$ are definable, we speak of definably Fréchet differentiability relative to $A$.

2000 Mathematics Subject Classification: Primary 03C64; Secondary 14P99, 26B05, $58 \mathrm{C} 20$.

Key words and phrases: o-minimal structure, Fréchet derivative, extensions.

Research partially supported by NSERC Discovery Grant of Dr. Salma Kuhlmann and by EC-IHP-Network RAAG (Contract-No: HPRN-CT-2001-00271). 
Recall that a function $f$ is of class Baire 1 if and only if $f$ is the pointwise limit of a sequence of continuous functions. The extendibility of Fréchet differentiable functions was discussed in [1], whose main result is the subsequent theorem. The symbol $\nabla$ denotes the gradient operator.

Theorem 1.2 (Aversa, Laczkovich and Preiss). Let $A \subset \mathbb{R}^{n}$ be closed and let $f: A \rightarrow \mathbb{R}$ together with $\varphi: A \rightarrow \mathbb{R}^{n}$ be Fréchet differentiable relative to $A$. Then there is a Fréchet differentiable function $F: \mathbb{R}^{n} \rightarrow \mathbb{R}$ with $\left.F\right|_{A}=f$ and $\left.\nabla F\right|_{A}=\varphi$ if and only if $\varphi$ is of class Baire 1.

We study and prove an o-minimal version of the above theorem which reads as follows.

Theorem 1.3. Let $A \subset R^{n}$ be a closed definable set, and let $f: A \rightarrow R$ together with $\varphi: A \rightarrow R^{n}$ be definably Fréchet differentiable. Then there is a definable Fréchet differentiable function $F: R^{n} \rightarrow R$ such that $\left.F\right|_{A}=f$ and $\left.\nabla F\right|_{A}=\varphi$.

Note that in the o-minimal version, the Baire 1 property of the derivative is not needed. Theorem 1.3 implies the next one. For $a, b \in R^{n}$ the Euclidean scalar product of $a$ and $b$ is denoted by $a \cdot b$.

Theorem 1.4. Let $f: A \rightarrow R$ be a definable function with closed domain such that for each $a \in A$ there is an element $d \in R^{n}$ satisfying

$$
\lim _{A \ni x \rightarrow a} \frac{f(x)-f(a)-d \cdot(x-a)}{\|x-a\|}=0 .
$$

Then $f$ is the restriction of a definable Fréchet differentiable function $F$ : $R^{n} \rightarrow R$.

Note that in the situation described in Theorem 1.4, definability of the set $A$ is needed. There exist closed (non-definable) subsets $A$ of $\mathbb{R}^{n}$ and (non-definable) functions $f: A \rightarrow \mathbb{R}$ which satisfy (1.2) but which cannot be extended as Fréchet differentiable functions to $\mathbb{R}^{n}$ (cf. [1]).

We strengthen the concept of Baire 1 functions as follows. A function $f: A \rightarrow R^{k}$ is of class definably Baire 1 if there is a definable family of continuous functions $F:(0,1) \times A \rightarrow R^{k}$ such that $f(a)=\lim _{t / 1} F(t, a)$, $a \in A$.

As a further application of Theorem 1.3 we obtain the statement below.

TheOrem 1.5. Every definable function $g$ is of class definably Baire 1.

There are two sections subsequent to the Introduction. The first is devoted to the preparation of the proof of Theorem 1.3, while in the last section we prove Theorems 1.3, 1.4 and 1.5. 
2. Preliminaries. We first study the extendibility on closures of special sets which we call $\mathcal{C}_{\text {Lip }}^{m}$ cells.

If $U$ and $V$ are definable sets and $m \in \mathbb{N}$, we use $\mathcal{C}^{m}(U, V)$ to denote the definable $m$ times continuously differentiable functions from $U$ to $V$; $\mathcal{C}_{\text {Lip }}^{m}(U, V)$ are the Lipschitz continuous elements of $\mathcal{C}^{m}(U, V)$. We generalise the notion of continuous differentiability to functions with non-open domain. A definable function $f: A \rightarrow R$ is called a $\mathcal{C}^{m}$ function if there exists a definable open neighbourhood $U$ of $A$ and a $\mathcal{C}^{m}$ function $F: U \rightarrow R$ such that $\left.F\right|_{A}=f$. The concept of $\mathcal{C}_{\text {Lip }}^{m}$ functions is analogously generalised to non-open domains.

Furthermore, $\pm \infty$ are regarded as constant functions defined on arbitrary sets.

Definition 2.1. A $\mathcal{C}_{\text {Lip }}^{m}$ cell in $R$ is either a single point or an open interval. Granted that we know the $\mathcal{C}_{\text {Lip }}^{m}$ cells in $R^{n-1}$, then a $\mathcal{C}_{\text {Lip }}^{m}$ cell in $R^{n}$ is either a single point, or a set $M$ of either the form

$$
M=\{(x, y): x \in X, y=h(x)\}
$$

where $X \subset R^{n-1}$ is a $\mathcal{C}_{\text {Lip }}^{m}$ cell and $h \in \mathcal{C}_{\text {Lip }}^{m}(X, R)$, or of the form

$$
M=\{(x, y): x \in X, f(x)<y<g(x)\}
$$

where $X \subset R^{n-1}$ is an open $\mathcal{C}_{\text {Lip }}^{m}$ cell and $f, g \in \mathcal{C}_{\text {Lip }}^{m}(X, R) \cup\{ \pm \infty\}$ are such that for all $x \in X, f(x)<g(x)$.

If $\alpha \in \mathbb{N}^{n}$ we denote by $D_{\alpha}$ the differential operator assigning to a sufficiently smooth mapping $f$ its $\alpha$ th derivative.

For the proof of the next lemma, approximation of definable $\mathcal{C}^{k}$ functions by $\mathcal{C}^{k+l}$ functions is required. This is provided by Escribano's approximation theorem which is proved in [8].

Theorem 2.2 (Escribano). Let $k \geq 0$ and $l \geq 1$ be integers, and let $X \subset R^{n}$ be definable and open. Let $f \in \mathcal{C}^{k}(X, R)$. Then, for every definable continuous function $\varepsilon: X \rightarrow(0, \infty)$, there is a $g \in \mathcal{C}^{k+l}(X, R)$ such that for all $\alpha \in \mathbb{N}^{n}$ with $\alpha_{1}+\cdots+\alpha_{n} \leq k$,

$$
\left|D_{\alpha} f(x)-D_{\alpha} g(x)\right|<\varepsilon(x), \quad x \in X .
$$

If $A \subset R^{d}$ where $d<n$, we let $A \times\{0\}:=\left\{(a, 0, \ldots, 0) \in R^{n}: a \in A\right\}$, and for a function $f: A \times\{0\} \rightarrow R^{k}$ we write $f(a, 0)$ instead of $f(a, 0, \ldots, 0)$, $a \in A$.

If $X$ is a definable set, we denote by $\operatorname{cl}(X)$ its topological closure and by $\partial X:=\operatorname{cl}(X) \backslash X$ its frontier.

Next we show the extendibility of definable Fréchet differentiable functions defined on the closure of certain kinds of $\mathcal{C}_{\text {Lip }}^{m}$ cells. 
Lemma 2.3. Let $d<n$ be a positive integer, $X \subset R^{d}$ be an open $\mathcal{C}_{\mathrm{Lip}}^{m}$ cell and let $f: \operatorname{cl}(X) \times\{0\} \rightarrow R$ together with $\varphi: \operatorname{cl}(X) \times\{0\} \rightarrow R^{n}$ be definably Fréchet differentiable relative to $\operatorname{cl}(X)$. If

(1) $X \ni x \mapsto f(x, 0)$ and $X \ni x \mapsto \varphi(x, 0)$ are continuously differentiable,

(2) $f(x, 0)=0$ and $\varphi(x, 0)=0, x \in \partial X$,

then, for every definable open neighbourhood $U$ of $X$, there is a definable Fréchet differentiable function $F: R^{n} \rightarrow R$ with the following properties:

(a) $\operatorname{supp}(F) \subset \operatorname{cl}(U)$,

(b) $F$ is $\mathcal{C}^{3}$ outside $\operatorname{cl}(X) \times\{0\}$,

(c) $F(x, 0)=f(x, 0)$ and $\nabla F(x, 0)=\varphi(x, 0), x \in X$.

Proof. Step 1. Let $(x, y)=\left(x_{1}, \ldots, x_{d}, y_{d+1}, \ldots, y_{n}\right) \in X \times R^{n-d}$, and let $G: X \times R^{n-d} \rightarrow R$ be given by

$$
G(x, y):=f(x, 0)+\sum_{i=d+1}^{n} \varphi_{i}(x, 0) y_{i} .
$$

Property (1) implies that $G$ is continuously differentiable and, by construction, $G$ is definable. In addition,

$$
G(x, 0)=f(x, 0) \quad \text { and } \quad \nabla G(x, 0)=\varphi(x, 0), \quad x \in X .
$$

STEP 2. Let $Z:=X \times\left(R^{n-d} \backslash\{0\}\right)$, and let $\widetilde{\varepsilon}: Z \rightarrow(0, \infty)$ be the definable continuous function defined by $\widetilde{\varepsilon}(x, y)=\min \left(1,\|y\|^{2}\right)$. We apply Theorem 2.2 to $G$ restricted to $Z$ with $k=1, l=2$ and $\varepsilon=\widetilde{\varepsilon} / \sqrt{n}$, so that we obtain a $\mathcal{C}^{3}$ function $H: Z \rightarrow R$ which satisfies the inequalities

$$
|(H-G)(z)| \leq \varepsilon(z) \quad \text { and } \quad|\nabla(H-G)(z)| \leq \widetilde{\varepsilon}(z), \quad z \in Z .
$$

Let dist $(-,-)$ denote the Euclidean distance function where we allow sets to be the second argument. We further select a function $\varrho \in \mathcal{C}^{3}\left(R^{d},[0, \infty)\right)$ which vanishes outside $X$ and which satisfies for every $x \in X$ the inequality

$$
0<\varrho(x)<\min \left(1, \frac{\operatorname{dist}(x, \partial X)^{2}}{1+|f(x, 0)|+\sum_{i=0}^{n}\left|\varphi_{i}(x)\right|}, \frac{1}{2} \operatorname{dist}((x, 0), \partial U)\right) .
$$

Further let $\psi \in \mathcal{C}^{3}([0, \infty),[0,1])$ be equal to 1 on $[0,1 / 2]$ and 0 on $[1, \infty)$.

Step 3. We define $F: R^{n} \rightarrow R$ by

$$
F(x, y):= \begin{cases}H(x, y) \psi(\|y\| / \varrho(x)), & (x, y) \in Z \\ G(x, 0), & (x, 0) \in X \times\{0\} \\ 0, & \text { otherwise }\end{cases}
$$


Then $F$ is definable by construction. Let

$$
B:=\{(x, y): x \in X,\|y\|<\varrho(x)\} .
$$

Then $F$ vanishes outside $B$ so that property (a) is evident.

The choice of $\psi$ and $\varrho$ implies that $F$ is $\mathcal{C}^{3}$ smooth in $Z$; obviously, it is also $\mathcal{C}^{3}$ in the complement of $\operatorname{cl}(B)$. Thus, $F$ is $\mathcal{C}^{3}$ outside $\operatorname{cl}(X) \times\{0\}$, which proves property (b).

Note that $|F(x, y)-G(x, y)| \leq\|y\|^{2}$ whenever $(x, y) \in B$. Therefore, $F-G$ is Fréchet differentiable in $B$ and both $F-G$ and $\nabla(F-G)$ vanish in $X \times\{0\}$. This implies (c).

It remains to prove the Fréchet differentiability of $F$ at every point of $\partial X \times\{0\}$. For $(x, y) \in B,\|y\|$ is bounded by $\varrho(x)$, and both $\varepsilon$ and $\psi$ are bounded by 1 . Hence,

$$
\begin{aligned}
|F(x, y)| & \leq|G(x, y)|(1+\varepsilon(x, y)) \psi\left(\frac{\|y\|}{\varrho(x)}\right) \leq 2|G(x, y)| \\
& \leq 2|f(x, 0)|+2 \sum_{i=d+1}^{n}\left|\varphi_{i}(x, 0) y_{i}\right| \\
& \leq 2|f(x, 0)|+2 \sum_{i=d+1}^{n}\left|\varphi_{i}(x, 0) \varrho(x)\right| \\
& \leq 2|f(x, 0)|+2 \operatorname{dist}(x, \partial X)^{2} .
\end{aligned}
$$

For every $z \in \partial X$, property (2) now implies that

$$
\lim _{(x, y) \rightarrow(z, 0)} \frac{f(x, y)}{\|(x, y)-(z, 0)\|}=0 .
$$

So,

$$
\lim _{(x, y) \rightarrow(z, 0)} \frac{|F(x, y)|}{\|(x, y)-(z, 0)\|} \leq \lim _{(x, y) \rightarrow(z, 0)} \frac{2 \operatorname{dist}(x, \partial X)^{2}}{\|(x, y)-(z, 0)\|}=0 .
$$

Therefore, $F$ is Fréchet differentiable at all points of $\partial X \times\{0\}$.

The derivatives of Fréchet differentiable functions defined on non-open sets are in general not unique, which calls for a generalised version of the chain rule. In this connection, definability is not required.

Lemma 2.4 (Chain rule). Let $X \subset R^{n}$ and $Y \subset R^{m}$. Let $f: X \rightarrow Y$ together with $\varphi: X \rightarrow R^{n \times m}$ and $g: Y \rightarrow R^{k}$ together with $\gamma: Y \rightarrow R^{m \times k}$ be Fréchet differentiable functions relative to $X$ respectively $Y$. Then $h:=$ $g \circ f: X \rightarrow R^{p}$ together with $\theta: X \rightarrow R^{n \times k}$ is Fréchet differentiable relative to $X$, where

$$
\theta(x):=\gamma(f(x)) \varphi(x), \quad x \in X
$$


Proof. We fix $x \in X$ and set $\xi:=f(x)$. By assumption, there are functions $\phi_{f}: X \rightarrow R^{m}$ and $\phi_{g}: Y \rightarrow Z$ such that for all $y \in X, \eta \in Y$,

$$
\begin{gathered}
f(y)-f(x)=\varphi(x)(y-x)+\phi_{f}(y-x), \\
g(\eta)-g(\xi)=\gamma(\xi)(\eta-\xi)+\phi_{g}(\eta-\xi),
\end{gathered}
$$

and

$$
\lim _{y \rightarrow x} \frac{\phi_{f}(y-x)}{\|y-x\|}=0, \quad \lim _{\eta \rightarrow \xi} \frac{\phi_{g}(\eta-\xi)}{\|\eta-\xi\|}=0 .
$$

Let $\varepsilon>0$. We select $\delta>0$ so small that for any $y \in X$ with $\|y-x\|<\delta$,

$$
\begin{aligned}
& \left\|\phi_{g}\left(\varphi(x)(y-x)+\phi_{f}(y-x)\right)\right\|<\varepsilon\|y-x\| / 2, \\
& \|\gamma(\xi)\|\left\|\phi_{f}(y-x)\right\| \leq \varepsilon\|y-x\| / 2 .
\end{aligned}
$$

Then

$$
\begin{aligned}
\| h(y) & -h(x)-\theta(x)(y-x)\|=\| g(\eta)-g(\xi)-\gamma(\xi) \varphi(x)(y-x) \| \\
& =\left\|g(\eta)-g(\xi)-\gamma(\xi)\left(f(y)-f(x)+\phi_{f}(y-x)\right)\right\| \\
& \leq\left\|\phi_{g}(f(y)-f(x))\right\|+\|\gamma(\xi)\|\left\|\phi_{f}(y-x)\right\| \\
& \leq\left\|\phi_{g}\left(\varphi(x)(y-x)+\phi_{f}(y-x)\right)\right\|+\varepsilon\|y-x\| / 2 \leq \varepsilon\|y-x\| .
\end{aligned}
$$

Hence, $h$ is Fréchet differentiable at $x$ relative to $X$ with formal derivative $\theta(x)$.

If $\lambda$ is a $\mathcal{C}_{\text {Lip }}^{1}$ function, note that $\lambda$ extends to a (uniquely determined) definable Lipschitz continuous function $\bar{\lambda}$ with domain $\operatorname{cl}(X)$.

Lemma 2.5. Let $Y$ be a $\mathcal{C}_{\text {Lip }}^{3}$ cell and $U$ be a definable open neighbourhood of $Y$. Let $f: \operatorname{cl}(Y) \rightarrow R$ together with $\varphi: \operatorname{cl}(Y) \rightarrow R$ be a definable Fréchet differentiable function relative to $\operatorname{cl}(Y)$ such that

(1) $f$ and $\varphi$ are $\mathcal{C}^{2}$ functions in $Y$,

(2) $f(\eta)=0$ and $\varphi(\eta)=0$ for $\eta \in \partial Y$.

Then there is a definable Fréchet differentiable function $F: R^{n} \rightarrow R$ such that

(a) $\operatorname{supp}(F) \subset \operatorname{cl}(U)$,

(b) $F$ is $\mathcal{C}^{3}$ outside $\mathrm{cl}(Y)$, and

(c) $F(\eta)=f(\eta), \nabla F(\eta)=\varphi(\eta)$, for $\eta \in \operatorname{cl}(Y)$.

Proof. If $Y$ is an open set, we set $\left.F\right|_{Y}=f$ and $\left.F\right|_{R^{n} \backslash Y}=0$.

If $Y$ consists of a single point $y$, then $\partial Y=\emptyset$. We select a $\mathcal{C}^{3}$ function $\psi: R \rightarrow R$ which vanishes outside $(-1,1)$ and equals 1 in $(-1 / 2,1 / 2)$. We further select an $\varepsilon>0$ such that the ball with radius $\varepsilon$ and centre $y$ is 
contained in $U$. Then the definable function $F: R^{n} \rightarrow R$ given by

$$
F(x):=\psi\left(1-\frac{1}{\varepsilon}\|x-y\|^{3}\right)\left(f(y)+\sum_{i=1}^{n} \varphi_{i}(y)\left(x_{i}-y_{i}\right)\right)
$$

satisfies the conclusion of this lemma.

In all other cases, after some permutation of variables, we may assume that $Y=(h)_{X}:=\{(x, y): x \in X, y=h(x)\}$ where $X \subset R^{d}$ is an open definable $\mathcal{C}_{\text {Lip }}^{3}$ cell and $h: X \rightarrow R^{n-d}$ a definable $\mathcal{C}_{\text {Lip }}^{3}$ mapping.

We define $\lambda: X \times R^{n-d} \rightarrow X \times R^{n-d}$ by $\lambda(x, y):=(x, y+h(x))$. So $\lambda$ is a $\mathcal{C}_{\text {Lip }}^{3}$ function with $\mathcal{C}_{\text {Lip }}^{3}$ smooth inverse. Therefore, both $\lambda$ and $\lambda^{-1}$ extend continuously to definable Lipschitz continuous functions defined on the closure of $X \times R^{n-d}$. The bijectivity of both functions is preserved.

SteP 1: Reduction to the situation of Lemma 2.3. We define $g$ : $\operatorname{cl}(X) \times\{0\} \rightarrow R$ and $\gamma: \operatorname{cl}(X) \times\{0\} \rightarrow R^{n}$ by

$$
\begin{aligned}
g(x, 0) & :=f(\lambda(x, 0)), \quad x \in \operatorname{cl}(X), \\
\gamma(x, 0) & :=(\varphi(\lambda(x, 0))) \nabla \lambda(x, 0), \quad x \in X,
\end{aligned}
$$

and $\gamma(x, 0):=0$ for $x \in \partial X$.

Firstly, since $\lambda$ is Fréchet differentiable in $Y$, we obtain Fréchet differentiability of $g$ together with $\gamma$ relative to $X \times\{0\}$ by Lemma 2.4.

Secondly, $\bar{\lambda}$ is bijective. Let $L$ be a Lipschitz constant for both $\bar{\lambda}$ and $\bar{\lambda}^{-1}$. Then, for $\xi \in \partial X \times\{0\}$,

$$
\begin{aligned}
\lim _{X \times\{0\} \ni x \rightarrow \xi} \frac{|g(x)|}{\|x-\xi\|} & =\lim _{X \times\{0\} \ni x \rightarrow \xi} \frac{|f(\bar{\lambda}(x))|}{\|x-\xi\|} \\
& \leq \lim _{X \times\{0\} \ni x \rightarrow \xi} \frac{L|f(\bar{\lambda}(x))|}{\|\bar{\lambda}(x)-\bar{\lambda}(\xi)\|} \\
& \leq \lim _{Y \ni \bar{\lambda}(x) \rightarrow \bar{\lambda}(\xi)} \frac{L|f(\bar{\lambda}(x))|}{\|\bar{\lambda}(x)-\bar{\lambda}(\xi)\|}=0 .
\end{aligned}
$$

Thus, $g$ together with $\gamma$ is Fréchet differentiable relative to $\operatorname{cl}(X) \times\{0\}$.

Both $\lambda$ and $\varphi$ are $\mathcal{C}^{2}$ functions so that $\gamma$ is $\mathcal{C}^{1}$ in $X$. Therefore, $g$ together with $\gamma$ satisfies the conditions of Lemma 2.3 .

Step 2. According to Lemma 2.3 there exists a definable Fréchet differentiable function $G: R^{n} \rightarrow R$ such that

$$
\begin{aligned}
& \operatorname{supp}(G) \subset \operatorname{cl}(W), \\
& G \text { is } \mathcal{C}^{3} \text { in } R^{n} \backslash(\operatorname{cl}(X) \times\{0\}), \\
& G(x, 0)=g(x, 0), \nabla G(x, 0)=\gamma(x, 0), x \in X .
\end{aligned}
$$


We now define $F: R^{n} \rightarrow R$ by

$$
F(z)= \begin{cases}G\left(\lambda^{-1}(z)\right), & z \in \lambda(W) \\ 0, & \text { otherwise }\end{cases}
$$

STEP 3: Verifying properties (a)-(c). The support of $F$ is a subset of $\operatorname{cl}(\lambda(W)) \subset \operatorname{cl}(U)$, and therefore (a) follows.

The function $F$ is $\mathcal{C}^{3}$ in $R^{n} \backslash \operatorname{cl}(\lambda(W))$ and in $\left(X \times R^{n-d}\right) \backslash \operatorname{cl}(Y)$. Hence, $F$ is $\mathcal{C}^{3}$ outside $\operatorname{cl}(Y)$, which proves property $(\mathrm{b})$.

Of course, $F$ is Fréchet differentiable at all $y \in Y$, and, by Lemma 2.4,

$$
\nabla F(y)=\varphi\left(\lambda\left(\lambda^{-1}(y)\right)\right)(\nabla \lambda)\left(\nabla \lambda^{-1}\right)=\varphi(y)
$$

Moreover, for $\eta \in \partial Y$,

$$
\begin{aligned}
\lim _{\eta \neq y \rightarrow \eta} \frac{|F(y)|}{\|y-\eta\|} & =\lim _{\eta \neq y \rightarrow \eta} \frac{\left|G\left(\bar{\lambda}^{-1}(y)\right)\right|}{\|y-\eta\|} \leq \lim _{\eta \neq y \rightarrow \eta} \frac{L\left|G\left(\bar{\lambda}^{-1}(y)\right)\right|}{\left\|\bar{\lambda}^{-1}(y)-\bar{\lambda}^{-1}(\eta)\right\|} \\
& \leq \bar{\lambda}_{\bar{\lambda}^{-1}(\eta) \neq \bar{\lambda}^{-1}(y) \rightarrow \bar{\lambda}^{-1}(\eta)} \frac{L\left|G\left(\bar{\lambda}^{-1}(y)\right)\right|}{\left\|\bar{\lambda}^{-1}(y)-\bar{\lambda}^{-1}(\eta)\right\|}=0 .
\end{aligned}
$$

Hence, $F$ is Fréchet differentiable at all points of $\operatorname{cl}(Y)$, that is, (c) is evident.

3. Proofs. The proof of Theorem 1.3 involves the concept of $\mathcal{C}_{\text {Lip }}^{m}$ stratification which we introduce next.

Definition 3.1. Let $m$ be a positive integer. A $\mathcal{C}_{\text {Lip }}^{m}$ stratification of a closed definable subset $A \subset R^{n}$ is a finite partition of $A$ into subsets $S_{1}, \ldots, S_{r}$, called strata, with the following properties.

(1) For each $i$ there is a linear orthogonal isomorphism $\Phi_{i}: R^{n} \rightarrow R^{n}$ such that $\Phi_{i}\left(S_{i}\right)$ is a $\mathcal{C}_{\text {Lip }}^{m}$ cell.

(2) For each stratum $S$, the frontier $\partial S$ is the union of some strata.

A stratification $S_{1}, \ldots, S_{r}$ of $A$ is called compatible with the subsets $A_{1}, \ldots, A_{k}$ of $A$ if each $A_{j}$ is the union of some of the strata, $j=1, \ldots, k$.

For any definable sets $A_{1}, \ldots, A_{k} \subset R^{n}$ there is a $\mathcal{C}_{\text {Lip }}^{m}$ stratification compatible with $A_{1}, \ldots, A_{k}$. This is implied by the stronger concept of $\Lambda^{m}$ regular stratification (cf. [10, Theorem 1.4]).

Since every $\mathcal{C}_{\text {Lip }}^{m}$ cell is a $\mathcal{C}^{m}$ cell (cf. [4, Chapter 7.3]), each $\mathcal{C}_{\text {Lip }}^{m}$ cell in $R^{n}$ is definably homeomorphic to $R^{d}$ for a unique $0 \leq d \leq n$ (cf. [2, Lemma 2.6]). The number $d$ is called the dimension of the cell. For an arbitrary definable set $X$, the dimension of $X$ is given by

$$
\operatorname{dim}(X)=\max \{d: X \text { contains a set }
$$

definably homeomorphic to $R^{d}$ \}. 
A detailed discussion of dimension in o-minimal structures can be found in [4, Chapter 4]. We need the fundamental relation between the dimension of a definable set and its frontier. If $X$ is definable, then, by [4, Chapter 4 , Theorem 1.8],

$$
\operatorname{dim}(\partial X)<\operatorname{dim}(X)
$$

This fact, in connection with property (2) in the definition of stratification, implies that for each stratum $S$ there is a definable open neighbourhood $U$ of $S$, which is disjoint from every stratum $S^{\prime} \neq S$ with $\operatorname{dim}\left(S^{\prime}\right) \leq$ $\operatorname{dim}(S)$.

A further interesting property of a definable function $f$ is that the domain can be partitioned into finitely many definable sets such that the restriction of $f$ to any of these sets is a $\mathcal{C}^{m}$ function (cf. [4, Chapter 7.3]).

Proof of Theorem 1.3. By using [10, Theorem 1.4] we select a $\mathcal{C}_{\text {Lip }}^{3}$ stratification of $R^{n}$ which is compatible with $A$ and the sets on which both $f$ and $\varphi$ are $\mathcal{C}^{2}$ functions. Furthermore, we order the strata $S_{1}, \ldots, S_{r}$ which are contained in $A$ in such a way that $\operatorname{dim}\left(S_{j}\right) \leq \operatorname{dim}\left(S_{j+1}\right), j=$ $1, \ldots, r-1$.

We proceed by induction on the number of cells $r$ contained in $A$.

If $r=1$, we obtain the statement of the theorem by Lemma 2.5.

For the induction step, we set $B:=\bigcup_{i=1}^{r-1} S_{i}$. Because of inequality (3.2) and as $B$ contains all strata $S \subset A$ of dimension less than $\operatorname{dim}(B)$, the set $B$ is closed. By the induction hypothesis, there exists a definable Fréchet differentiable function $H: R^{n} \rightarrow R$ which is $\mathcal{C}^{2}$ outside $B$ and which satisfies $\left.H\right|_{B}=\left.f\right|_{B}$ and $\left.\nabla H\right|_{B}=\left.\varphi\right|_{B}$.

Let $g: \operatorname{cl}\left(S_{r}\right) \rightarrow R$ and $\gamma: \operatorname{cl}\left(S_{r}\right) \rightarrow R^{n}$ be defined by $g(a)=f(a)-H(a)$ and $\gamma(a)=\varphi(a)-\nabla H(a), a \in \operatorname{cl}\left(S_{r}\right)$. The function $g$ together with $\gamma$ satisfies the conditions of Lemma 2.5. According to the properties of a stratification there is a definable open neighbourhood $U$ of $S_{r}$ disjoint from $B$. Lemma 2.5 yields a function $G$ which satisfies $\left.G\right|_{S_{r}}=g,\left.\nabla G\right|_{S_{r}}=\gamma$, and $G=0$ outside $U$. Then $F=G+H$ is a definable function satisfying the conclusions of Theorem 1.3.

In o-minimal structures, the following statement, known as definable choice (cf. [4, Chapter 6, Proposition 1.2]), holds true:

If $S \subset R^{m+n}$ is definable and $\pi: R^{m+n} \rightarrow R^{m}$ denotes the projection onto the first $m$ coordinates, then there is a definable function $f: \pi(S) \rightarrow R^{n}$ such that $\Gamma(f) \subset S$.

Proof of Theorem 1.4. Consider the set

$$
V:=\{(a, d): f \text { is Fréchet differentiable at } a \text { with derivative } d\} .
$$


Let $\Gamma(f)$ be the graph of $f$, and let $\Phi(a, d)$ be the formula

$$
\begin{aligned}
& a \in A \wedge \forall \varepsilon \in R[\varepsilon>0 \rightarrow \exists \delta[\delta>0 \rightarrow \forall x \in A \\
& {\left[\sum_{i=1}^{n}\left(x_{i}-a_{i}\right)^{2}<\delta^{2} \rightarrow \forall x \in R \forall y \in R\right.} \\
& {[[(y, a) \in \Gamma(f) \wedge(z, x) \in \Gamma(f)] \rightarrow} \\
& \left.\left.\left.\left.\left(z-y-\sum_{i=1}^{n} d_{i}\left(x_{i}-a_{i}\right)\right)^{2} \leq \varepsilon^{2} \sum_{i=1}^{n}\left(x_{i}-a_{i}\right)^{2}\right]\right]\right]\right] .
\end{aligned}
$$

Then $\Phi(a, d)$ is a first-order formula in the language of the o-minimal structure $\mathcal{M}$ (see [3, Chapter 1.3]). In addition, an element $(a, d) \in A \times R^{n}$ belongs to $V$ if and only if $\Phi(a, d)$ holds true. By [3, Theorem 1.13], $V$ is definable.

If $\pi: V \rightarrow A$ denotes the projection onto the first $n$ coordinates, then by applying definable choice to $V$ and $A=\pi(V)$ we obtain a definable function $\varphi: A \rightarrow R^{n}$ such that $\Gamma(\varphi) \subset V$. Hence, $f$ together with $\varphi$ is definably Fréchet differentiable relative to the closed definable set $A$. By Theorem 1.3, there is a definable Fréchet differentiable function $F: R^{n} \rightarrow R$ with $\left.F\right|_{A}=f$.

Proof of Theorem 1.5. Let $A \subset R^{n}$ be definable and let $g: A \rightarrow R$ be an arbitrary definable function.

We define $\varphi: \operatorname{cl}(A) \times\{0\} \rightarrow R^{n+1}$ by

$$
\varphi(a, 0):= \begin{cases}(0, \ldots, 0, g(a)) & \text { if } a \in A, \\ 0 & \text { if } a \in \partial A .\end{cases}
$$

If $f: \operatorname{cl}(A) \times\{0\} \rightarrow R$ is the zero function, then $f$ together with $\varphi$ is definably Fréchet differentiable relative to the definable closed set $\operatorname{cl}(A) \times\{0\}$. By applying Theorem 1.3 to $f$ and $\varphi$, there is a definable Fréchet differentiable function $F: R^{n+1} \rightarrow R$ such that $\left.\nabla F\right|_{\operatorname{cl}(A) \times\{0\}}=\varphi$. As $g(a)=\varphi_{n+1}(a, 0)$ for $a \in A$, and as Fréchet derivatives are of class definably Baire 1, the statement of the theorem is evident.

\section{References}

[1] V. Aversa, M. Laczkovich and D. Preiss, Extension of differentiable functions, Comment. Math. Univ. Carolin. 26 (1985), 597-609.

[2] A. Berarducci and M. Otero, Intersection theory for o-minimal manifolds, Ann. Pure Appl. Logic 107 (2001), 87-119.

[3] M. Coste, An Introduction to o-minimal Geometry, Dip. Mat. Univ. Pisa, Dottorato di Ricerca in Matematica, Istituti Editoriali e Poligrafici Internazionali, Pisa, 2000. 
[4] L. van den Dries, Tame Topology and o-minimal Structures, London Math. Soc Lecture Notes Ser. 248, Cambridge Univ. Press, 1998.

[5] L. van den Dries and C. Miller, Geometric categories and o-minimal structures, Duke Math. J. 84 (1996), 497-540.

[6] L. van den Dries and P. Speissegger, The real field with convergent generalized power series, Trans. Amer. Math. Soc. 350 (1998) 4377-4421.

[7] - - - The field of reals with multisummable series and the exponential function, Proc. London Math. Soc. (3) 81 (2000), 513-565.

[8] J. Escribano, Approximation theorems in o-minimal structures, Illinois J. Math. 46 (2002), 111-128.

[9] A. Fischer, Peano-differentiable functions in o-minimal structures, doctoral thesis, Universität Passau, 2006.

[10] -, o-minimal $\Lambda^{m}$-regular stratification, Ann. Pure Appl. Logic, online 2007.

[11] T. Kaiser, J.-P. Rolin and P. Speissegger, Transition maps at non-resonant hyperbolic singularities are o-minimal, preprint, Univ. of Regensburg, 2006.

[12] C. Miller, Expansions of the real field with power functions, Ann. Pure Appl. Logic 68 (1994), 79-94.

[13] C. Miller and L. van den Dries, On the real exponential field with restricted analytic functions, Israel J. Math. 85 (1994), 19-56.

[14] J.-P. Rolin, P. Speissegger and A. J. Wilkie, Quasianalytic Denjoy-Carleman classes and o-minimality, J. Amer. Math. Soc. 16 (2003), 751-777.

[15] A. J. Wilkie, Model completeness results for expansions of the ordered field of real numbers by restricted Pfaffian functions and the exponential function, ibid. 9 (1996), 1051-1094.

Department of Mathematics \& Statistics

University of Saskatchewan

106 Wiggins Road

Saskatoon, SK, Canada S7N 5E6

E-mail: el.fischerandreas@web.de

Received 22.2.2007

and in final form 14.5.2007 